\title{
葸类衍生物的电荷传输性质
}

\author{
段桂花 ${ }^{1}$ 高洪泽 ${ }^{2}$ 王丽娟 ${ }^{1}$ 张厚玉 ${ }^{1, *}$ 马於光 ${ }^{1, *}$ \\ ( ${ }^{1}$ 吉林大学超分子结构与材料国家重点实验室, 长春 $130012 ; 2$ 中国人民武装警察部队学院基础部, 河北 廊坊 065000)
}

\begin{abstract}
摘要：以具有较高迁移率的对称取代类蒽的衍生物 $\{2,6$ 二 二[2-(4-戊基苯基)乙烯基]葱, DPPVAnt; 2,6-二噻吩 蒽, DTAnt; 2,6-二[2-己基噻吩]蒽, DHTAnt \} 为研究对象, 采用密度泛函理论的 B3LYP 方法, 在 6-31G $(d)$ 的基组 水平上研究了三种蒽类衍生物的分子结构、电子结构、重组能和电荷传输积分, 采用 Einstein 关系式计算了室温 下的载流子迁移率, 并与蒽的相关计算结果进行了比较. DPPVAnt 是较好的空穴传输材料, 其空穴迁移率为 $0.49 \mathrm{~cm}^{2} \cdot \mathrm{V}^{-1} \cdot \mathrm{s}^{-1}$; DHTAnt 有利于电子传输, 其电子迁移率为 $0.12 \mathrm{~cm}^{2} \cdot \mathrm{V}^{-1} \cdot \mathrm{s}^{-1}$; 而 DTAnt 是一种较好的双极性材 料, 其空穴迁移率和电子迁移率分别为 0.069 和 $0.060 \mathrm{~cm}^{2} \cdot \mathrm{V}^{-1} \cdot \mathrm{s}^{-1}$. 计算得到的迁移率与实验结果处于同一数量 级. 三种葱类衍生物的电子重组能与葸的相近, 而空穴重组能均大于蒽的空穴重组能, 大小顺序为蒽 $<$ DPPVAnt $<$ DTAnt $<$ DHTAnt. 这与计算的迁移率结果不一致, 说明分子的堆积结构决定材料的电荷传输性质.
\end{abstract}

关键词：密度泛函理论；葸类衍生物；迁移率；电荷传输；分子重组能 中图分类号：0641

\section{Charge Transport Properties of Anthracene Derivatives}

\author{
DUAN Gui-Hua $\quad$ GAO Hong-Ze ${ }^{2} \quad$ WANG Li-Juan ${ }^{1} \quad$ ZHANG Hou-Yu ${ }^{1, *} \quad$ MA Yu-Guang ${ }^{1, *}$ \\ ('State Key Laboratory of Supramolecular Structure and Materials, Jilin University, Changchun 130012, P. R. China; \\ ${ }^{2}$ Fundamental Department, Chinese People's Armed Police Force Academy, Langfang 065000 , Hebei Province, P. R. China)
}

\begin{abstract}
The molecular geometries, electronic structures, reorganization energies, and charge transfer integrals of three anthracene derivatives \{2,6-bis [2-(4-pentylphenyl)vinyl]anthracene, DPPVAnt; 2,6-bis-thiophene anthracene, DTAnt; 2,6-bis[2-hexylthiophene]anthracene, DHTAnt $\}$ were investigated by density functional theory at the B3LYP/ 6-31G $(d)$ level. Their mobilities at room temperature were estimated using Einstein relations and compared with the calculated mobility of anthracene. DPPVAnt is a good hole-transporting material with a hole mobility as high as 0.49 $\mathrm{cm}^{2} \cdot \mathrm{V}^{-1} \cdot \mathrm{s}^{-1}$; DHTAnt is an electron-transporting material with an electron mobility of about $0.12 \mathrm{~cm}^{2} \cdot \mathrm{V}^{-1} \cdot \mathrm{s}^{-1}$; DTAnt is a bipolar material with its hole and electron mobilities being 0.069 and $0.060 \mathrm{~cm}^{2} \cdot \mathrm{V}^{-1} \cdot \mathrm{s}^{-1}$, respectively. The calculated mobilities were of the same magnitude as those obtained by experimental measurements. The reorganization energies for the electrons of the three derivatives are almost the same as that for anthracene but the reorganization energies for the holes of the three derivatives are larger than that of anthracene and they follow the order: anthracene $<$ DPPVAnt $<$ DTAnt $<$ DHTAnt. This is not in agreement with the order of the calculated mobilities and implies that the mobilities are determined by molecular packing.
\end{abstract}

Key Words: Density functional theory; Anthracene derivative; Mobility; Charge transport; Molecular reorganization energy

近年来, 随着有机半导体材料在有机电致发光 发的不断深人, 人们逐渐认识到影响有机半导体电 二极管、薄膜晶体管、太阳能电池和激光器等领域研 子器件性能的一个重要参数是载流子的迁移率 ${ }^{[1-5]}$.

Received: February 22, 2010; Revised: April 21, 2010; Published on Web: June 11, 2010.

*Corresponding authors. Email: houyuzhang @jlu.edu.cn, ygma@jlu.edu.cn.

The project was supported by the National Natural Science Foundation of China (20603013) and National Key Basic Research Program of China (973) (2009CB623605).

国家自然科学基金(20603013)和国家重点基础研究发展规划项目(973) (2009CB623605)资助

(C) Editorial office of Acta Physico-Chimica Sinica 
迄今为止, 在薄膜晶体管中应用最为广泛的是并五 苯及其衍生物, 其场效应迁移率高达 $3.0 \mathrm{~cm}^{2} \cdot \mathrm{V}^{-1} \cdot \mathrm{s}^{-1[6]}$. 但是, 以并五苯为基础的有机薄膜晶体管材料的空 气稳定性非常有限, 影响器件的寿命. 利用葸具有较 宽带隙的特点来提高材料的稳定性, Meng 等 ${ }^{[7-8]}$ 合 成了三种对称取代的葸的衍生物 \{2,6-二[2-(4-戊基 苯基)乙烯基]葱, DPPVAnt; 2,6-二-噻吩葱, DTAnt; 2,6-二[2-已基噻吩]葱, DHTAnt $\}$, 其优化后的结构 示意图如图 1 所示), 且这些衍生物具有较高的载流 子迁移率. 载流子迁移率在很大程度上依赖于实验 测定的条件和方法, 而理论模拟可以研究有机半导 体材料中电荷输运机制和计算材料的本征载流子迁 移率, 能够从更微观的角度去理解、印证和预测实 验的测量数据. Deng 等 ${ }^{[9]}$ 采用 Marcus-Hush 两态模 型的方法, 估算了蒽晶体中分子间的电荷转移积分, 并由此预测了葸的空穴载流子迁移率为 $1.84 \mathrm{~cm}^{2}$. $\mathrm{V}^{-1} \cdot \mathrm{s}^{-1}$. Kukhta 等 ${ }^{[00]}$ 研究了葱类衍生物的离子势、电 子亲合势和分子重组能等性质. 目前对于葱类衍生 物的载流子传输性质, 特别是葱类衍生物的分子结 构如何影响迁移率的理论研究还不是很深人, 报道 也很少见

本文以三种蒽类衍生物为主要研究对象, 分析 影响三个化合物电荷传输的因素, 寻找电荷的传输 路径, 并通过计算其重组能和传输积分, 预测室温条 件下的迁移率, 并与同样方法计算得到的葱的载流 子迁移性质作比较进行分析, 为这三种葋类衍生物 的实际应用提供理论基础.

\section{1 理论基础与计算方法}

对于有序的有机半导体材料的电荷传输, 在低 温下采用准能带的模型, 通过有效质量的方法来计
算空穴和电子的迁移率. 而在室温或更高温度条件 下, 温度的升高带来热致无序化效应和散射, 使能带 的模型不再适用, 通常采用电荷在分子间跳跃的机 制来描述 ${ }^{[4,9,11-12]}$

由 Einstein 方程式可知, 迁移率 $\mu$ 与扩散系数 $D$ 的关系可以表示为 ${ }^{[0]}$

$$
\mu=\frac{e}{k_{\mathrm{B}} T} D ; \quad D=\frac{1}{6} \sum_{i} r^{2} P_{i} W_{i}
$$

式(1)中, $T$ 为室温 $(298 \mathrm{~K}), e$ 为电子电荷, $k_{\mathrm{B}}$ 为玻尔 兹曼常数, $D$ 为电荷以一个分子为起点向三维空间 方向上的扩散系数, $P_{i}$ 是电荷向第 $i$ 个相邻分子迁 移的几率, $P_{i}=W_{i} / \sum_{i} W_{i}, r$ 为相邻两分子的质心距离, $W_{i}$ 是电荷向第 $i$ 个相邻分子的迁移速率, 用 Marcus 电荷转移理论表示为 ${ }^{[13]}$ :

$$
W=\frac{2 \pi V^{2}}{h}\left(\frac{\pi}{\lambda k_{\mathrm{B}} T}\right)^{1 / 2} \exp \left(-\frac{\lambda}{4 k_{\mathrm{B}} T}\right)
$$

式(2)中, $V$ 为传输积分, $h$ 为 Planck 常数, $\lambda$ 为分子 的重组能. 要得到较大的迁移率, 就要求物质具有尽 可能小的重组能和尽可能大的传输积分.

重组能 $\lambda$ 主要由内重组能和外重组能两部分 组成 ${ }^{[14-17]}$. 在晶体状态或无定形薄膜下载流子传输 的实验和理论研究表明, 外重组能的贡献很小 ${ }^{[18-19]}$, 本文对其忽略不计. 分子的内重组能 $\lambda$ 是从中性态 到离子态的几何弛豫能 $\left(\lambda_{\mathrm{rel}}^{(1)}\right)$ 和与之相反过程的弛豫 能 $\left(\lambda_{\mathrm{rel}}^{(2)}\right)$ 两部分之和, 即

$$
\lambda=\lambda_{\text {rel }}^{(1)}+\lambda_{\text {rel }}^{(2)}=\left[E^{+}\left(G^{0}\right)-E^{+}\left(G^{+}\right)\right]+\left[E^{0}\left(G^{+}\right)-E^{0}\left(G^{0}\right)\right]
$$
式(3)中, $E^{+}\left(G^{0}\right)$ 和 $E^{0}\left(G^{0}\right)$ 为优化的中性分子构型下带 一个正电荷和不带电荷时的能量, 而 $E^{+}\left(G^{+}\right)$和 $E^{0}\left(G^{+}\right)$ 为优化的带正电荷的分子构型下带一个正电荷和不 带电荷的能量. 上式中 $E^{+}$和 $G^{+}$的上角标正号换成负

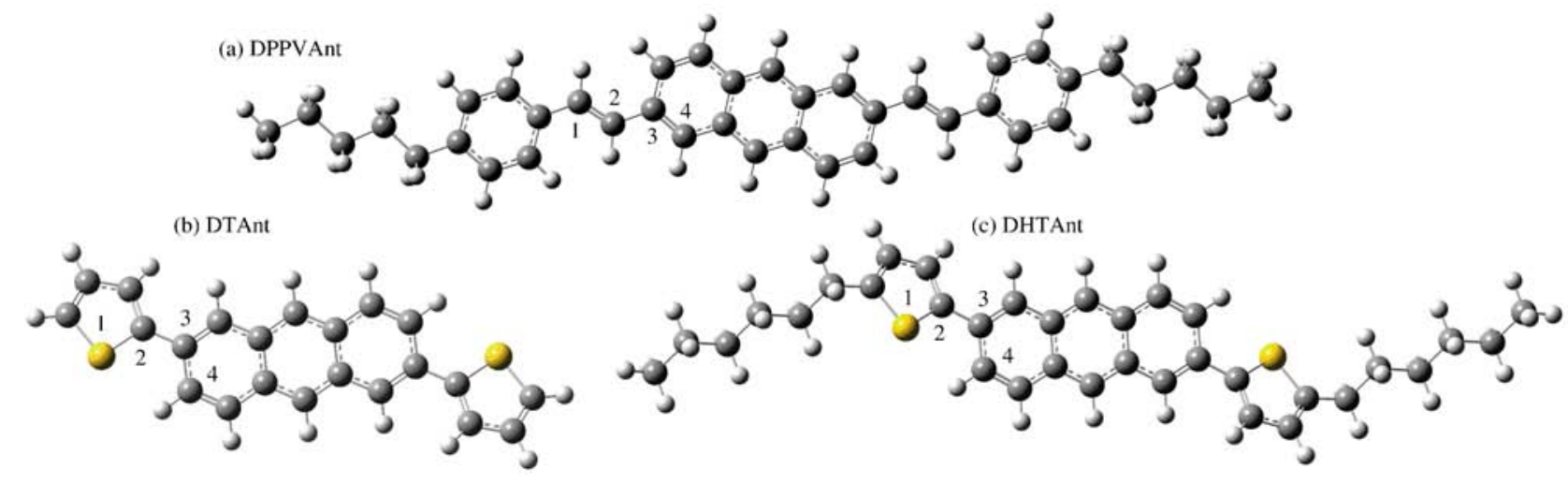

图 1 三种葸类衍生物中性态下优化的分子结构

Fig.1 Optimized structures of the three anthracene derivatives in the neutral state 
号, 就可以计算分子得到一个电子时的重组能.

传输积分 $V$ 的计算方法主要有: 应用 Koopman 定理的间接法 ${ }^{[20-22]}$, 直接耦合法 ${ }^{[23-26]}$, 两态模型下的 变分方法 ${ }^{[27-29]}$, 分割技术方法 ${ }^{[30-32]}$, 以及应用在光诱 导电子转移体系的广义 Mulliken-Hush 方法 ${ }^{[33-37}$ 等. 本文采用直接法求传输积分, 可以用下式求算:

$$
V=\left\langle\Psi_{i}^{0, \mathrm{Ml}}|\mathbf{F}| \Psi_{j}^{0, \mathrm{M} 2}\right\rangle
$$

式(4)中, $\Psi_{i}^{0, \mathrm{M} 1}, \Psi_{j}^{0, \mathrm{M} 2}$ 分别为两个邻近分子 $\mathrm{M} 1, \mathrm{M} 2$ 的 前线分子轨道, 上角标 0 表示没有微扰混合前的轨 道; $\mathbf{F}$ 为二聚体分子的单电子 Fock 算符, 可通过 $\mathbf{F}=$ $S c \varepsilon c^{-1}$ 计算得到, 其中, $S$ 表示邻近两个分子形成的 二聚体的重叠矩阵, $\boldsymbol{c}$ 和 $\boldsymbol{\varepsilon}$ 分别为轨道系数矩阵和 能量矩阵. 传输空穴(电子)时, $i, j$ 表示邻近分子的 HOMO(LUMO) 轨道, HOMO 代表最高占有轨道, LUMO 代表最低空轨道.

全部计算采用密度泛函理论 ${ }^{[38-39]}$ 的 B3LYP/6$31 \mathrm{G}(d)$ 方法 ${ }^{[0-41]}$, 用 Gaussian 09 软件包 ${ }^{[42]}$ 完成. 传输 积分的数据处理采用自编的矩阵运算程序完成.

\section{2 结果与讨论}

\section{1 分子结构和重组能}

三种葱类衍生物中性态的优化结构如图 1 所 示. 中性态、阳离子态和阴离子态的部分键长和二面 角参数的计算值和实验值 ${ }^{[7-8]}$ 见表 1 . 计算得到的分 子的重组能列于表 2. 与晶体结构相比, 中性态的 DPPVAnt 中乙烯单元与葱更趋于平面, 而 DTAnt 和 DHTAnt中的噻吩环与葸具有更大的扭转角. 这 是由于晶体结构中分子排列受限的结果. 与 DPPVAnt 中性态的优化结构相比, 它的离子态的几 何结构变化不大, $\mathrm{C} 2-\mathrm{C} 3$ 键长缩短 $0.002 \mathrm{~nm}$, 而 $\mathrm{C} 1-\mathrm{C} 2$ 键长增长 $0.002 \mathrm{~nm}$, 阳离子的构型与中性 态构型更为接近, 阴离子的分子结构变化更大些, 有 更好的平面性. 分子结构的变化与表 2 中计算的分

表 1 葸类衍生物在中性态和离子态下的部分优化结构参数 Table 1 Selected optimized geometry parameters of anthracene derivatives in the neutral and ionic states

\begin{tabular}{cllll}
\hline Compd. & \multicolumn{1}{c}{ Parameter } & \multicolumn{1}{c}{ Neutral } & Cationic & Anionic \\
\hline DPPVAnt & $R(\mathrm{C} 2-\mathrm{C} 3) / \mathrm{nm}$ & $0.146\left(0.144^{[7]}\right)$ & 0.144 & 0.144 \\
& $R(\mathrm{C} 1-\mathrm{C} 2) / \mathrm{nm}$ & $0.135\left(0.132^{[7]}\right)$ & 0.137 & 0.137 \\
& $\theta(\mathrm{C} 1 \mathrm{C} 2 \mathrm{C} 3 \mathrm{C} 4) /\left(^{\circ}\right)$ & $179.9\left(165.6^{[7]}\right)$ & 179.9 & 180.0 \\
DTAnt & $R(\mathrm{C} 2-\mathrm{C} 3) / \mathrm{nm}$ & $0.147\left(0.147^{[7]}\right)$ & 0.144 & 0.145 \\
& $\theta(\mathrm{S} 1 \mathrm{C} 2 \mathrm{C} 3 \mathrm{C} 4) /\left(^{\circ}\right)$ & $28.1\left(9.0^{[8]}\right)$ & 0.2 & 16.5 \\
DHTAnt & $R(\mathrm{C} 2-\mathrm{C} 3) /\left(^{\circ}\right)$ & $0.147\left(0.149^{[8]}\right)$ & 0.144 & 0.145 \\
& $\theta(\mathrm{S} 1 \mathrm{C} 2 \mathrm{C} 3 \mathrm{C} 4) /\left(^{\circ}\right)$ & $26.9\left(2.4^{[8]}\right)$ & 0.5 & 16.5 \\
\hline
\end{tabular}

The data in the parentheses are the experimental values.
表 2 蒽及其三种衍生物的空穴和电子重组能

Table 2 Reorganization energies of anthracene and its three derivatives for hole and electron

\begin{tabular}{lll}
\hline \multirow{2}{*}{ Compd. } & \multicolumn{2}{c}{ Reorganization energy $(\mathrm{eV})$} \\
\cline { 2 - 3 } & \multicolumn{1}{c}{ hole } & \multicolumn{1}{c}{ electron } \\
\hline anthracene & $0.137\left(0.135^{[10]}\right)$ & $0.198\left(0.194^{[10]}\right)$ \\
DPPVAnt & 0.181 & 0.204 \\
DTAnt & 0.261 & 0.202 \\
DHTAnt & 0.288 & 0.205 \\
\hline
\end{tabular}

子重组能是一致的. DPPVAnt 的空穴和电子重组能 分别为 0.181 和 $0.204 \mathrm{eV}$, 空穴和电子重组能相近, 而电子重组能稍大. 而 DTAnt 和 DHTAnt 的离子态 几何构型与中性态构型相比变化较大, 并且阳离子 构型要比阴离子构型变化大, 尤其是噻吩环与葱环 的二面角, 这些结构变化表明它们的空穴重组能会 大于电子重组能, 这也与计算的重组能结果是一致 的, DTAnt 和 DHTAnt 的空穴重组能大于电子重组 能. 计算得到的葱类衍生物的空穴和电子重组能分 别为 0.137 和 $0.198 \mathrm{eV}$, 与文献的报道 ${ }^{[10]}$ 吻合. 三种 葱类衍生物的电子重组能相近, 稍大于葸的电子 重组能, 而空穴重组能均远大于葸的空穴重组能, 大小顺序为 DPPVAnt $<$ DTAnt $<$ DHTAnt. 仅从重组 能对迁移率影响的角度来看, 三种衍生物与葸相比 在重组能上对传输空穴没有优势. 相比而言, 化合物 DPPVAnt 具有较小的空穴重组能, 更有利于空穴传 输, 而化合物 DTAnt 和DHTAnt 不利于空穴传输. 三 种衍生物具有相近的电子重组能, 在传输电子方面 能力也应相近. 影响葸及其衍生物载流子的传输性 能的另一因素是分子间的传输积分的大小, 迁移率 需要由这两个因素来共同决定.

\section{2 分子的前线轨道}

载流子传输与前线分子轨道分布密切相关 ${ }^{[43]}$, 电子的传输是通过分子间的 LUMO 传递的, 而空穴 传输是通过分子间 HOMO 传递的. 因而前线分子 轨道电子云的分布以及离域程度的大小都会影响 电荷的传输. 三种衍生物的前线轨道如图 2 所示. 三 种衍生物的 HOMO 和 LUMO 几乎全部分布在葱环 及两端与之共轭的苯乙烯或噻吩环上, 但 HOMO 和 LUMO 分布也有区别. 对于 DPPVAnt, 其 LUMO 的 离域性明显小于HOMO 的, 特别是两端的苯环上, 电 子云零散地分布在 4 个 $\mathrm{C}$ 上; 对于 DTAnt, 其 LUMO 和 HOMO分布比较接近. DHTAnt 的 LUMO 的离域 性会比 DTAnt 的大. DPPVAnt 分子的离域长度最 长, DHTAnt 次之, DTAnt 最短. 从前线分子轨道的 


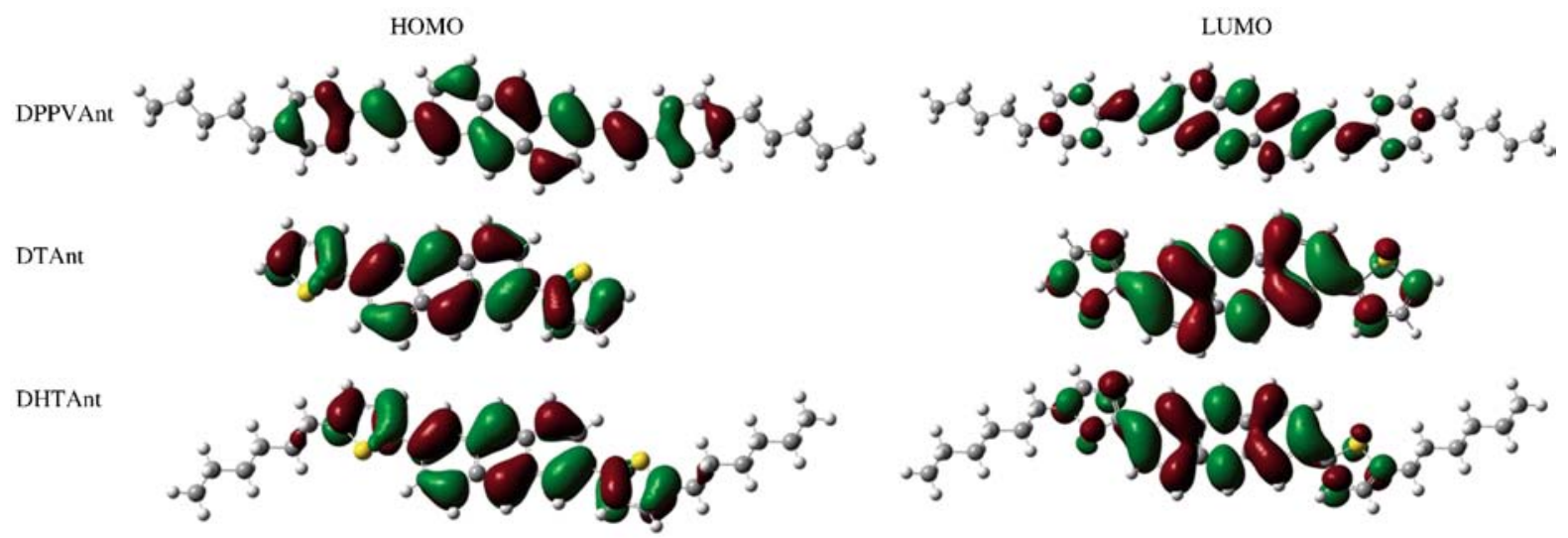

图 2 三种葸类衍生物中性态下分子的前线轨道图

Fig.2 HOMOs and LUMOs plots of the three anthracene derivatives in the neutral state

HOMO: the highest occupied molecular orbital; LUMO: the lowest unoccupied molecular orbital

离域程度的角度可以预测, DPPVAnt 的空穴传输能 力会大于其电子传输能力; DTAnt 很可能是较好的 双极性传输材料, 空穴和电子的传输会比较平衡; 而 DHTAnt 相对于 DTAnt 会有利于电子的传输.

\section{3 传输积分及迁移率}

要计算分子间的电荷传输积分, 必须确定电荷 的传输路径. 对于有机材料, 在器件中主要以无定形 态存在, 随着实验手段的进步, 薄膜越来越有序, 在 几十到几百纳米尺度下都可以看成是以有序的晶体 形式进行堆积 ${ }^{[4]}$. 不同的晶体结构就近似代表了薄膜 的不同存在形态. 我们以分子的晶体结构 ${ }^{[7-8]}$ 确定分 子在空间的相对位置, 来计算分子的传输积分. 选择 晶体中的一个分子作为载流子的给体, 该分子向周 围每一个邻近分子的传输作为一种传输路径. 三种 葸类衍生物和葸分子中主要的传输路径如图 3 所 示, 计算得到的电子和空穴的传输积分值以及相应 的计算和实验 ${ }^{[7,8,45]}$ 迁移率见表 3 .

三种葱类衍生物的晶体堆积密度都大于并五 苯和葱, 其中 DPPVAnt 的堆积密度最大(结构参数 $a, b$ 的大小列于表 $4, a, b$ 方向见图 3). 三种蒽类衍 生物中同层内的分子间距离参数 $a$ 和隔层间距离 参数 $b$ 都比对应的并五苯 ${ }^{[46]}$ 的小. 与葱相比, 三种
葱衍生物中参数 $a$ 与葸的结构参数 ${ }^{[47]}$ 相近, 但参数 $b$ 比蒽的小. DHTAnt 的堆积也比 DTAnt 的堆积紧 密. 晶体呈现 “人字形”排列, 与葸相似. DPPVAnt 和 DHTAnt 的较大密度堆积是分子共轭芳香环之间 $\pi-\pi$ 相互作用和两端烷基链之间较强的范德华相 互作用力共同作用的结果. 以 DPPVAnt 晶体为例, 沿着分子长轴方向看, 以一个分子为中心, 与周围 6 个分子存在紧密的相互作用(见图 3), 其中 4 种为分 子间等距离的分子边对面的 “人字形”相互作用, 为 不同层间分子相互作用(如图 4(a)所示). 2 种为同层 分子之间等距离的错位平行相互作用(图 4(b)所示). 分子沿着长轴方向头尾相接的传输途径为 8 种, 但 是质心间距离较远, 分子间相互作用较弱. 所以分子 中载流子的传输途径是沿着分子短轴方向为主, 具 有明显的各向异性的特点.

三种葸类衍生物与葸的电荷传输路径基本相 同. 以 DPPVAnt 为例, 两种主要的传输路径中的轨 道相互作用如图 4. 所示. 无论是“人字形”还是错位 平行的排列中, 空穴的相互作用都远大于电子的相 互作用, 电荷传输积分值见表 3 . 利用式(1), 室温下 计算得到 DPPVAnt 的空穴和电子迁移率分别是 0.49 和 $0.042 \mathrm{~cm}^{2} \cdot \mathrm{V}^{-1} \cdot \mathrm{s}^{-1}$. 空穴的载流子迁移率比电

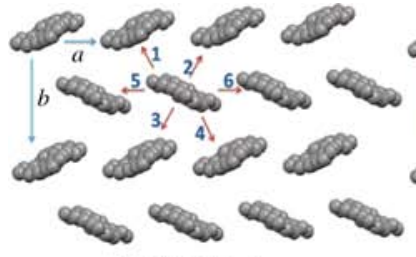

(a) DPPVAnt

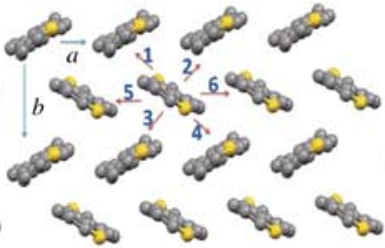

(b) DTAnt

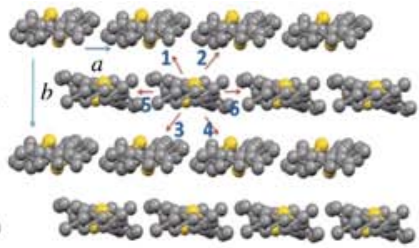

(c) DHTAnt

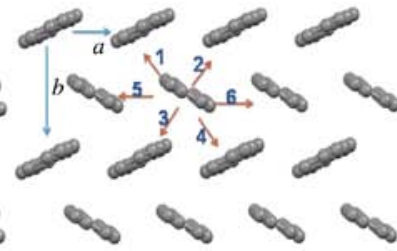

(d) anthracene

图 3 葱及其三种衍生物晶体结构中主要的传输路径

Fig.3 Main pathways in the crystal structures of anthracene and its three derivatives 
表 3 三种葱类衍生物电子和空穴的传输积分和迁移率

Table 3 Transfer integrals and mobilities of the three anthracene derivatives for hole and electron

\begin{tabular}{|c|c|c|c|c|c|c|c|}
\hline \multirow{2}{*}{ Compd. } & \multirow{2}{*}{ Pathway } & \multirow{2}{*}{ Distance (nm) } & \multicolumn{2}{|c|}{$10^{5} \mathrm{~V} / \mathrm{eV}$} & \multicolumn{3}{|c|}{$\mu /\left(\mathrm{cm}^{2} \cdot \mathrm{V}^{-1} \cdot \mathrm{s}^{-1}\right)$} \\
\hline & & & hole & electron & hole & electron & expt. \\
\hline \multirow[t]{6}{*}{ DPPVAnt } & 1 & 0.465 & 7430.8 & 2456.6 & 0.49 & 0.042 & $0.1-1.28^{[7]}$ \\
\hline & 2 & 0.465 & 7430.9 & 2457.6 & & & \\
\hline & 3 & 0.465 & 7431.2 & 2457.8 & & & \\
\hline & 4 & 0.465 & 7430.3 & 2456.3 & & & \\
\hline & 5 & 0.585 & 3039.8 & 1919.4 & & & \\
\hline & 6 & 0.585 & 3039.7 & 1917.2 & & & \\
\hline \multirow[t]{6}{*}{ DTAnt } & 1 & 0.483 & 4183.6 & 2594.3 & 0.069 & 0.060 & $0.037^{[8]}$ \\
\hline & 2 & 0.483 & 4183.2 & 2594.3 & & & \\
\hline & 3 & 0.483 & 4183.6 & 2594.3 & & & \\
\hline & 4 & 0.483 & 4183.2 & 2594.3 & & & \\
\hline & 5 & 0.609 & 887.2 & 2457.8 & & & \\
\hline & 6 & 0.609 & 887.2 & 2457.8 & & & \\
\hline \multirow[t]{6}{*}{ DHTAnt } & 1 & 0.472 & 3860.9 & 4086.2 & 0.038 & 0.12 & $0.13^{[8]}$ \\
\hline & 2 & 0.472 & 3862.3 & 4086.8 & & & \\
\hline & 3 & 0.472 & 3860.9 & 4086.2 & & & \\
\hline & 4 & 0.472 & 3858.2 & 4086.5 & & & \\
\hline & 5 & 0.587 & 2282.8 & 2990.2 & & & \\
\hline & 6 & 0.587 & 2283.2 & 2990.9 & & & \\
\hline \multirow[t]{6}{*}{ anthracene } & 1 & 0.516 & 3000.9 & 5052.5 & 0.25 & 0.22 & $0.57-2.07^{[45]}$ \\
\hline & 2 & 0.516 & 3002.0 & 5052.3 & & & \\
\hline & 3 & 0.515 & 3001.7 & 5052.6 & & & \\
\hline & 4 & 0.516 & 3001.1 & 5052.2 & & & \\
\hline & 5 & 0.599 & 3595.2 & 2630.3 & & & \\
\hline & 6 & 0.599 & 3595.2 & 2630.3 & & & \\
\hline
\end{tabular}

子的载流子迁移率约高一个数量级, 表明 DPPVAnt 是较好的空穴传输材料. DTAnt 中分子平行排列更 有利于电子的传输, 计算得到的空穴迁移率和电子 迁移率分别为 0.069 和 $0.060 \mathrm{~cm}^{2} \cdot \mathrm{V}^{-1} \cdot \mathrm{s}^{-1}$, 二者非常 接近, 表明化合物 DTAnt 可能是一种较好的双极性 材料, 载流子的传输比较平衡; 而 DHTAnt 中两种 分子排列方式中电子的传输积分都大于空穴的传输 积分. 计算的电子迁移率和空穴迁移率分别达到 0.12 和 $0.038 \mathrm{~cm}^{2} \cdot \mathrm{V}^{-1} \cdot \mathrm{s}^{-1}$, 说明更有利于电子的传 输. 由此可见, 不同的取代基可以改变葸的传输性 质, 如 DPPVAnt 是以传输空穴为主, 而 DHTAnt 是 以传输电子为主. 计算得到的葱的空穴和电子的迁

表 4 蒽类衍生物以及并五苯和蒽的晶体结构参数

Table 4 Crystal structure parameters of anthracene derivatives, pentacene, and anthracene

\begin{tabular}{lcc}
\hline \multirow{2}{*}{ Compd. } & \multicolumn{2}{c}{ Parameter $(\mathrm{nm})$} \\
\cline { 2 - 3 } & $a$ & $b$ \\
\hline DPPVAnt & 0.585 & 0.723 \\
DTAnt & 0.609 & 0.751 \\
DHTAnt & 0.587 & 0.740 \\
pentacene $^{[46]}$ & 0.627 & 0.779 \\
anthracene $^{[4]]}$ & 0.599 & 0.841 \\
\hline
\end{tabular}

移率分别为 0.25 和 $0.22 \mathrm{~cm}^{2} \cdot \mathrm{V}^{-1} \cdot \mathrm{s}^{-1}$, 比文献的计 算值 ${ }^{[45]}$ 小, 可能是文献中采用两态模型计算会高估 电荷转移积分, 从而导致计算的迁移率偏大. 与实验 测得的总的载流子迁移率 ${ }^{[7-8,45]}$ 相比, 计算得到的迁 移率数值与实验测量值处于相同的数量级内. 计算 得到的载流子迁移率能从更微观的角度理解材料的

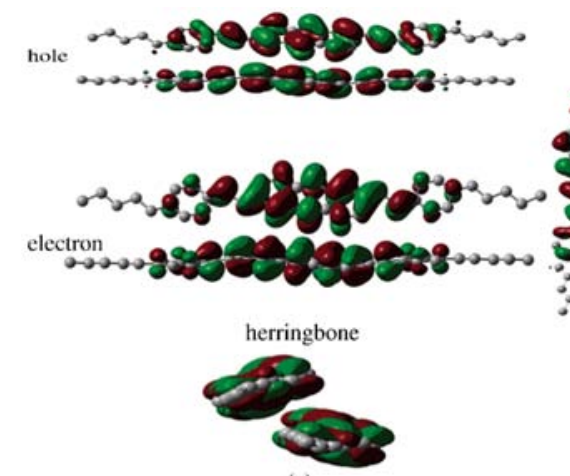

(a)

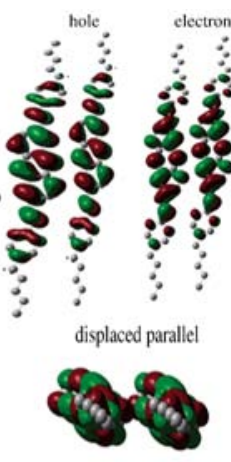

(b)
图 4 DPPVAnt 晶体的空穴和电子的两种主要传输路径 中分子间轨道相互作用

Fig.4 Intermolecular orbital interactions for two kinds of pathways of hole and electron in DPPVAnt crystal

(a) herringbone mode; (b) displaced parallel mode 
电荷迁移特性, 例如是否更有利于电子或空穴传输. 计算得出的载流子迁移率的规律与分子的重组能不 一致, 但与前线轨道的离域程度得到的结论基本一 致. 虽然葋类衍生物的分子重组能远大于葱的, 但是 葸类衍生物的分子的堆积密度比葸的大, 从而传输 积分的大小与葸相当, 计算得出的迁移率也与蒽在 同样的数量级内.

\section{3 结 论}

通过计算表明, 蒽类衍生物具有与葸和并五苯 一样较高的载流子迁移率, 计算与实验的结果相符. DPPVAnt 是较好的空穴传输材料; DHTAnt 有利于 电子传输; 而 DTAnt 是一种较好的双极性材料. 同 时可以看出,引人不同的取代基, 在提高材料的稳定 性的同时, 还可以实现从传输空穴到传输电子的转 变. 三种葸类衍生物的电子重组能相近, 而空穴重组 能均大于葸的空穴重组能, 其大小顺序为葸< DPPVAnt $<$ DTAnt $<$ DHTAnt. 这与计算的迁移率的结 果不一致, 说明分子的堆积结构决定了材料的电荷 传输的性质. 这类取代的葱类衍生物由于具有较强 的分子间相互作用, 分子间堆积比较紧密, 有利于载 流子传输.

\section{References}

1 Pope, K.; Swenberg, C. E. Electronic processes in organic crystals and polymers. 2nd ed. New York: Oxford University Press, 1999

2 Silinsh, E. A.; Capek, V. Organic molecular crystals: interaction, localtion, and transport phenomena. New York: AIP Press, 1994

3 Gershenson, M. E.; Podzorov, V.; Morpurgo, A. F. Rev. Mod. Phys., 2006, 78: 973

4 Coropceanu, V.; Cornil, J.; da Silva Filho, D. A.; Olivier, V.; Silbey, R.; Bredas, J. L. Chem. Rev., 2007, 107: 926

5 Shirota, Y.; Kageyama, H. Chem. Rev., 2007, 107: 953

6 Klauk, K.; Halik, M.; Zschieschang, U.; Schmid, G.; Radlik, W. J. Appl. Phys., 2002, 92: 5259

7 Meng, H.; Sun, F. P.; Goldfinger, M. B.; Jaycox, G. D.; Li, Z. G.; Marshall, W. J.; Blackman, G. S. J. Am. Chem. Soc., 2005, 127 : 2406

8 Meng, H.; Sun, F. P.; Goldfinger, M. B.; Gao, F.; Londono, D. J.; Marshal, W. J.; Blackman, G. S.; Dobbs, K. D.; Keys, D. E. J. Am. Chem. Soc., 2006, 128: 9304

9 Deng, W. Q.; Goddard III, W. A. J. Phys. Chem. B, 2004, 108: 8614

10 Kukhta, A. V.; Kukhta, I. N.; Kukhta, N. A.; Neyra, O. L.; Meza, E. J. Phys. B-At. Mol. Opt. Phys., 2008, 41: 205701

11 Yang, X. D.; Wang, L. J.; Wang, C. L.; Long, W.; Shuai, Z. G. Chem. Mater., 2008, 20: 3205

12 Wang, C. L.; Wang, F. H.; Yang, X. D.; Li, Q. K.; Shuai, Z. G.
Organic Electrons, 2008, 9: 635

13 Marcus, R. A. Rev. Mod. Phys., 1993, 65: 599

14 Marcus, R. A. J. Chem. Phys., 1965, 43: 679

15 Newton, M. D.; Sutin, N. Annu. Rev. Phys. Chem., 1984, 35: 437

16 Siders, P.; Marcus, R. A. J. Am. Chem. Soc., 1981, 103: 748

17 Brunschwig, B. S.; Logan, J.; Newton, M. D.; Sutin, N. J. Am. Chem. Soc., 1980, 102: 5798

18 Vilfan, I. Physica Status Solidi B-Basic Research, 1973, 59: 351

19 Norton, J. E.; Bredas, J. L. J. Am. Chem. Soc., 2008, 130: 12377

20 Hutchison, G. R.; Ratner, M. A.; Marks, T. J. J. Am. Chem. Soc. 2005, 127: 16866

21 Lin, B. C.; Cheng, C. P.; You, Z. Q.; Hsu, C. P. J. Am. Chem. Soc., 2005, 127: 66

22 Cornil, J.; Beljonne, D.; Calbert, J. P.; Brédas, J. L. Adv. Mater. 2001, 13: 1053

23 Yang, X. D.; Li, Q.; Shuai, Z. G. Nanotechnology, 2007, 18: 424029

24 Troisi, A.; Orlandi, G. Chem. Phys. Lett., 2001, 344: 509

25 Yin, S. W.; Yi, Y. P.; Li, Q. X.; Yu, G.; Liu, Y. Q.; Shuai, Z. G. J. Phys. Chem. A, 2006, 110: 7138

26 Gao, H. Z.; Qin, C. S.; Zhang, H. Y.; Wu, S. Y.; Su, Z. M.; Wang, Y. J. Phys. Chem. A, 2008, 112: 9097

27 Liang, C.; Newton, M. D. J. Phys. Chem., 1992, 97: 3199

28 Dogonzdze, R. R.; Kuznetsov, A. M.; Vorotyntsev, M. A. Physica Status Solidi B-Basic Research, 1972, 54: 425

29 Newton, M. D. Chem. Rev., 1991, 91: 767

30 Larsson, S. J. Am. Chem. Soc., 1981, 103: 4034

31 Löwdin, P. O. J. Mol. Spectrosc., 1963, 10: 12

32 Siddarth, P.; Marcus, R. A. J. Phys. Chem., 1990, 94: 2985

33 Hush, N. S. Electrochim. Acta, 1968, 13: 1005

34 Creutz, C.; Newton, M. D. J. Photoch. Photobio. A, 1994, 82: 47

35 Cave, R. J.; Newton, M. D. J. Chem. Phys., 1997, 106: 9213

36 Cave, R. J.; Newton, M. D. Chem. Phys. Lett., 1996, 249: 15

37 Kryachko, E. S. J. Phys. Chem. A, 1999, 103: 4368

38 Hohenberg, P.; Kohn, W. Phys. Rev., 1964, 136: B864

39 Kohn, W.; Sham, L. J. Phys. Rev., 1965, 140: Al133

40 Becke, A. D. J. Chem. Phys., 1993, 98: 5648

41 Lee, C.; Yang, W. T.; Parr, R. G. Phys. Rev. B, 1988, 37: 785

42 Frisch, M. J.; Trucks, G. W.; Schlegel, H. B.; et al. Gaussian 09 Revision A.02. Wallingford, CT: Gaussian Inc., 2009

43 Liao, Y.; Su, Z. M.; Chen, Y. G.; Kan, Y. H.; Duan, H. X.; Qiu, Y Q.; Wang, R. S. Chem. J. Chin. Univ., 2003, 24: 477 [廖 奕, 苏忠民, 陈亚光, 阚玉和, 段红霞, 仇永清, 王荣顺. 高等学校化学 学报, 2003, 24: 477]

44 Shuai, Z. G.; Shao, J. S. Theretical chemistry: principles and applications. Beijing: Science Press, 2008 [帅志刚, 邵久书。 理论化学: 原理和应用. 北京: 科学出版社, 2008]

45 Silinsh, E. A.; Capek, V. Organic molecular crystal: interaction, localization and transport phenomena. New York: AIP Press, 1994: 332-333

46 Stefan, T. B.; Marta, M. T.; Peter, H.; Concepcioó, R. J. Am. Chem. Soc., 2004, 126: 6544

47 Brock, C. P.; Dunitz, J. D. Acta Crystallogr. Sect. B-Struct. Sci., 1990, 46: 795 